\title{
Lithium and metal abundances in long period Am binaries
}

\author{
I.Kh. Iliev ${ }^{1,2}$, J. Budaj ${ }^{1}$, J. Zverko ${ }^{1}$, I.S. Barzova ${ }^{2}$, and J. Žižňovský ${ }^{1}$ \\ 1 Astronomical Institute, Slovak Academy of Sciences, 05960 Tatranská Lomnica, The Slovak Republic \\ e-mail (Internet): (budaj, zve, ziga)@ta3.sk \\ 2 Institute of Astronomy, National Astronomical Observatory, P.O.B. 136, BG-4700, Smolyan, Bulgaria \\ e-mail (Internet): rozhen@sm.unacs.bg
}

Received January 3; accepted August 20, 1997

\begin{abstract}
High signal-to-noise CCD spectra of three bright, long period Am binaries (HD 108651, HD 116657 and HD 138213) have been obtained and the atmospheric abundances of identified chemical elements were studied. We found HD 116657 to be a new candidate as a Li deficient, long period Am binary, in addition to 16 Ori. While HD 108651 and HD 116657 have pronounced Am characteristics, HD 138213 is just a mild Am star.

These stars were further studied in a more general context related to the role of binarity in $\mathrm{CP}$ phenomenon. It was found that both Li abundance and the equivalent with ratio Ca I 6718/Fe I 6679 are more peculiar in systems with more pronounced eccentricities. Such behaviour is the opposite of what was previously found in Ap binaries and their anomalies. It indicates that tidal effects are of crucial importance in driving $\mathrm{CP}$ phenomena.
\end{abstract}

Key words: stars: chemically peculiar - stars: abundances — stars: individual: HD 108651, HD 116657, HD 138213 - binaries: general

\section{Introduction}

Lithium always poses an interesting problem because of its unique sensitivity to various mixing processes in stellar envelopes. This is also the case on the hot side of the Li gap. Theoretical calculations of Li diffusion predict a $\mathrm{Li}$ cloud will form under the superficial convection zone. This should result in a Li bump - overabundance on the hot side of the Li gap at around $7000 \mathrm{~K}$ accompanied by a high Li deficit for hotter stars (Richer \& Michaud 1993). Modern CCD detectors enabled Boesgaard (1987), Burkhart et al. (1987) and Burkhart \& Coupry $(1989,1991,1997)$ to study Li in Am stars for the first time. They concluded

Send offprint requests to: J. Budaj

* Based on observations collected at the National Astronomical Observatory Rozhen, Bulgaria. that in general $\mathrm{Li}$ is normal in $\mathrm{Am}$ and normal $\mathrm{A}$ stars in the range $\log N(\mathrm{Li} / \mathrm{H})=3.1 \pm 0.3($ with $\log N(\mathrm{H})=12)$ with a tendency to a slight deficit in Am stars ${ }^{1}$. In addition, they found that the scatter of observed abundances is rather high, but that there might be a predominance of $\mathrm{Li}$ deficient Am stars among the cooler ones. The star 16 Ori is a unique exception as it has by far the largest lithium deficit among the Am stars, as well as large anomalies of other elements (Burkhart \& Coupry 1989). Having an orbital period $P_{\mathrm{orb}}=155^{\mathrm{d}}$ and eccentricity $e=0.67$ it belongs to a very rare group of long period Am binaries. The main aim of this paper is to explore $\mathrm{Li}$ in three other long period Am binaries, namely HD 108651, HD 116657 and HD 138213.

It is well known that Am stars occur in binary systems with a frequency much higher than that of normal stars (Abt 1961, 1965 and Abt \& Bidelman 1969). Recently Budaj (1994, 1996, 1997), pursuing further the pioneering ideas of Abt in Am binaries, showed that: the metallicity tends to increase with $P_{\text {orb }}$ up to at least $50^{\mathrm{d}}$, probably even up to $200^{\mathrm{d}}$; the maximum rotation velocity and the curve of constant metallicity depend on $P_{\text {orb }}$; and the orbital period distribution (OPD) revealed an interesting gap within $180-800^{\mathrm{d}}$. To account for these features he formulated the tidal mixing+stabilization hypothesis (consult the references above for more details), which assumes the tidal effects to stretch to large $P_{\text {orb }}$ 's and to play a dominant role in driving Am phenomena. The new hydrodynamical retardation mechanism of Tassoul \& Tassoul (1992) might well fit into this pattern. The other goal of this paper is to inspect the metal abundance anomalies (mainly $\mathrm{Ca}, \mathrm{Fe}$ ) of the long period Am binaries in the most questionable region of orbital periods ranging from 50 up to 200 days.

Finally, it is aimed to put our results, along with previously published data, in a more general context to look

\footnotetext{
1 Note that the solar abundance of lithium differs from the cosmic value $(\log N(\mathrm{Li} / \mathrm{H})+12=3.31$ as derived from meteorites) by about -2.15 dex (Grevesse \& Anders 1991).
} 
for further evidence of tidal effects in Am stars. Namely, we will study the suspected relation of lithium and calcium/iron abundances on the eccentricities and orbital periods of Am binaries.

\section{Observations and data reduction}

All observations were made with the 2-m RCC telescope of the Bulgarian National Astronomical Observatory during 1994 and 1996. The Third Camera of the coudé spectrograph and the $580 \times 520$ ISTA CCD matrix with a pixel size $24 \times 18 \mu \mathrm{m}$ were used. The entrance slit of $300 \mu \mathrm{m}$ corresponding to 0.8 arcsec was set to provide a spectral power of 30000 . With a $632 \mathrm{~mm}^{-1}$ Bausch \& Lomb grating, a spectral range of $6675-6725 \AA \AA$ was covered with a dispersion of $4.2 \AA \mathrm{mm}^{-1}$ and a resolution of $0.22 \AA$. No binning was used. The reference spectrum was provided by a hollow-cathode Fe-Ar lamp. Flat-field frames were made by using a tungsten lamp. A shortened version of the $\log$ book is presented in Table 1. The PC-based IPS software package (Smirnov et al. 1992) was used for the flatfield correction, normalization and wavelength calibration. Consequently, for each star, we applied the spectra coaddition technique proposed by Hill \& Adelman (1986). As a result the $\mathrm{S} / \mathrm{N}$ ratio of about 250 for HD 108651, 350 for HD 116657 and 300 for HD 138213 was reached at the $2 \sigma$ level.

Table 1. List of observations

\begin{tabular}{|l|r|l|l|}
\hline HD & Date & Time & Exp. \\
\hline 108561 & 3.3 .1996 & $23: 50$ & $7 \times 30$ \\
& 7.4 .1996 & $00: 10$ & $8 \times 30$ \\
& 31.5 .1996 & $19: 00$ & $2 \times 30$ \\
& 1.6 .1996 & $21: 00$ & $2 \times 30$ \\
& 26.7 .1996 & $19: 10$ & $2 \times 30$ \\
116657 & 31.7 .1994 & $18: 40$ & $1 \times 15$ \\
& 26.7 .1996 & $20: 10$ & $2 \times 20$ \\
& 27.7 .1996 & $20: 10$ & $2 \times 20$ \\
138213 & 30.7 .1994 & $19: 10$ & $2 \times 30$ \\
& 31.7 .1994 & $18: 50$ & $1 \times 30$ \\
& 25.7 .1996 & $23: 00$ & $3 \times 30$ \\
& 26.7 .1996 & $21: 30$ & $3 \times 30$ \\
& 27.7 .1996 & $21: 30$ & $3 \times 30$ \\
\hline
\end{tabular}

Note: Time is in UT and relates to the middle of an exposure.

\section{Atmospheric parameters, spectrum synthesis and results}

In Table 2 we summarize relevant information about our program stars. The $U B V$, uvby $\beta$ (de-reddened by using $U V B Y$ BETA code of Moon \& Dworetsky 1985) indices and $v \sin i$ values are from Renson (1991). Geneva pho- tometry is from Rufener (1980). Orbital periods and eccentricities of HD 108651, HD 116657 and HD 138213 are according to Conti \& Barker (1973), Gutmann (1965) and Lucy \& Sweeney (1971), respectively.

Table 2. Photometry and other important information about the observed stars

\begin{tabular}{|l|l|l|l|}
\hline Star & HD 108561 & HD 116657 & HD 138213 \\
& HR 4751 & HR 5055 & HD 5752 \\
\hline$U-B$ & 0.08 & 0.1 & 0.1 \\
$B-V$ & 0.22 & 0.13 & 0.1 \\
$V$ & 6.6 & 4.0 & 6.1 \\
\hline$E(b-y)$ & 0.006 & -0.01 & -0.014 \\
$(b-y)_{0}$ & 0.114 & 0.063 & 0.046 \\
$m_{0}$ & 0.233 & 0.239 & 0.191 \\
$c_{0}$ & 0.832 & 0.911 & 1.141 \\
$\beta$ & 2.843 & 2.886 & 2.86 \\
\hline$U$ & 1.516 & - & 1.648 \\
$V$ & 0.689 & - & 0.825 \\
$B 1$ & 0.958 & - & 0.909 \\
$B 2$ & 1.411 & - & 1.445 \\
$V 1$ & 1.400 & - & 1.524 \\
$G$ & 1.828 & - & 1.989 \\
\hline$P_{\text {orb }[\mathrm{d}]}$ & 68.29 & 175.55 & 105.95 \\
$e$ & 0.36 & 0.46 & 0.0 \\
$v \sin i\left[\mathrm{~km} \mathrm{~s}^{-1}\right]$ & 15 & 60 & 30 \\
\hline
\end{tabular}

Table 3 summarizes the atmospheric parameters as derived from the calibrations of different authors. Temperatures from $U B V$ photometry as well as those of Castelli \& Kurucz (1994) from $\beta$ index are all systematically lower compared with the other calibrations of Strömgren or Geneva photometry. Nevertheless, the most elaborated and two-dimensional calibrations of Smalley \& Dworetsky (1995) and Kobi \& North (1990) are both consistent. Thus, we accepted their rounded off means as the best choice for model atmosphere parameters. All three stars are SB1 binaries, hence the possible influence of their companions was neglected.

A detailed spectrum synthesis of the spectral region around the $\mathrm{Li}$ I 6708 line was accomplished using the SYNTH (Piskunov 1992) and SYNSPEC (Hubený 1987; Zboril 1989, private communication) codes with the model atmospheres interpolated from Kurucz (1992) ATLAS9 grid. VALD atomic line database was used to create a line list for the spectrum synthesis (Piskunov et al. 1995). We first estimated the iron abundance. However, the normal abundance of iron would result in a quite insufficient depth of the synthetic absorption profile at $6705 \AA$, while at $6713 \AA$ it would be deeper by more than a factor of two. Unspecified missing opacity at $6705 \AA$ was also mentioned by Gerbaldi et al. (1995) and Hack et al. (1997). Following one of the possibilities of how to cope with such an inconsistency (van't Veer 1997, private 
Table 3. Atmospheric parameters as obtained from different methods

\begin{tabular}{|l|l|l|l|l|l|c|}
\hline \multicolumn{2}{|c|}{ HD 108561 } & \multicolumn{2}{c|}{ HD 116657 } & \multicolumn{2}{c|}{ HD 138213 } & \multirow{2}{*}{ Note } \\
\hline$T_{\text {eff }}$ & $\log g$ & $T_{\text {eff }}$ & $\log g$ & $T_{\text {eff }}$ & $\log g$ & \\
\cline { 1 - 6 } $6980^{1}$ & - & $7770^{1}$ & - & $8070^{1}$ & - & U \\
$7550^{2}$ & - & $8090^{2}$ & - & $8290^{2}$ & - & \\
\hline & & & & & & \\
$7900^{1}$ & - & $8460^{1}$ & - & $8660^{1}$ & - & \\
$7770^{2}$ & - & $8260^{2}$ & - & $8470^{2}$ & - & S \\
$8130^{3}$ & $4.31^{3}$ & $8390^{3}$ & $4.4^{3}$ & $8430^{3}$ & $3.61^{3}$ & \\
$7800^{4,6}$ & - & $8150^{4,7}$ & - & $7900^{4,8}$ & - & \\
\hline & & & & & & \\
$7850^{1}$ & - & - & - & $8610^{1}$ & - & G \\
$7910^{5}$ & $4.17^{5}$ & - & - & $8410^{5}$ & $3.68^{5}$ & \\
\hline & & & & & & \\
8000 & 4.2 & 8400 & 4.4 & 8400 & 3.6 & A \\
\hline
\end{tabular}

Note: $\mathrm{U}, \mathrm{S}, \mathrm{G}$ - derived from $U B V$, Strömgren and Geneva photometry, respectively, A - adopted here.

${ }^{1}$-Hauck \& Künzli (1996), ${ }^{2}$-Napiwotzky et al. (1993),

${ }^{3}$-Smalley \& Dworetsky (1995), ${ }^{4}$-Castelli \& Kurucz (1994),

${ }^{5}$-Kobi \& North (1990) for $[M / H]=0$,

${ }^{6}$-for $\log g=4,{ }^{7}$-for $\log g=4.5,{ }^{8}$-for $\log g=3.5$.

communication), we adjusted $\log g f$ using the solar spectrum (Kurucz et al. 1984). For the solar photosphere we used a model with $T_{\text {eff }}=5777 \mathrm{~K}, \log g=4.437$, interpolated from Kurucz's (1992) grid. The value of microturbulence for the theoretical spectrum computation was taken $1 \mathrm{~km} \mathrm{~s}^{-1}$. Comparing the theoretical and observed solar equivalent widths we estimated the $\log g f$ values of the lines as follows: Fe I 6705.101, -1.10 (VALD -1.50); Fe I $6712.676,-4.62(-2.88)$ and Fe I 6713.046, -1.61 (-1.05). Moreover, two lines, Fe I 6713.745 and Fe I 6713.771 given in VALD seem to refer to the same transition as they have identical $\log g f$ values, lower excitation potentials, as well as lower and upper $J$ values. The difference of $0.026 \AA$ in wavelength is not unusual when referring to different sources. Accepting both the lines as different transitions, their $\log g f$ values would have to be less in order to reach a fit. In our line list only the latter one, $6713.771 \AA$, was retained. This also satisfied to the solar spectrum. Only after these adjustments could we fit the observed spectra of all three stars.

With the known Fe abundance we determined the microturbulent velocity value by fitting the profile of the blend at $6678 \AA$ created mainly by a stronger Fe I line which showed to be sensitive to $\xi_{\text {turb }}$. This allows the value to be set with a formal accuracy $\approx 0.1 \mathrm{~km} \mathrm{~s}^{-1}$ for a given abundance value. To derive the abundances of other elements, the computed spectra were convolved with the instrumental profile (Gaussian of $0.22 \AA$ half-width) and rotationally broadened to fit with the observed spectra. Though a good fit was reached for a majority of the ab- sorptions seen in the spectra (see Fig. 4), there are still a few features like $6691 \AA$ in HD 108651 or $6709 \AA$ in the two hotter stars which are not explained sufficiently with the derived abundances and known opacity sources.

The abundances obtained in this way are introduced in terms of $[N / H]=\log (N / H)_{\star}-\log (N / H)_{\odot}$ in Table 4 . Taking into account the accuracy of the atmospheric parameters, as well as atomic data, the values for $\mathrm{Li}, \mathrm{Al}$, $\mathrm{Si}, \mathrm{Ti}$ and $\mathrm{Fe}$ are determined within $\lesssim 0.2$ dex, while the abundances of the other elements, occurring mainly in weak blends, are only approximate.

Table 4. Abundances derived in terms of $[N / H]$ for our three stars. $\log A=\log \left(N_{\mathrm{el}} / N_{\mathrm{H}}\right)+12.00$

\begin{tabular}{|l|c|c|l|l|l|l|}
\hline & Sun & \multicolumn{3}{|c|}{$\mathrm{A}$} & \multicolumn{1}{c|}{$\mathrm{B}$} & \multicolumn{1}{c|}{$\mathrm{C}$} \\
\cline { 2 - 7 } & $\log A$ & 1 & 2 & 3 & 3 & 3 \\
\hline $\mathrm{Li}$ & 1.16 & & +1.79 & +1.92 & +1.64 & +2.06 \\
$\mathrm{C}$ & 8.60 & -0.27 & & -0.4 & -0.4 & -0.2 \\
$\mathrm{~N}$ & 8.00 & & & +0.5 & 0.0 & +0.3 \\
$\mathrm{Al}$ & 6.47 & -0.66 & & -0.81 & -0.49 & -0.02 \\
$\mathrm{Si}$ & 7.55 & +0.34 & & +0.53 & +0.45 & +0.67 \\
$\mathrm{Ca}$ & 6.36 & -0.25 & & $-0.37^{*}$ & $-0.33^{*}$ & $+0.12^{*}$ \\
$\mathrm{Ti}$ & 4.99 & +0.09 & & -0.04 & -0.04 & -0.04 \\
$\mathrm{Fe}$ & 7.67 & +0.07 & +0.15 & +0.19 & +0.01 & +0.08 \\
$\mathrm{Ni}$ & 6.25 & +0.59 & & +0.9 & & \\
$\mathrm{Ce}$ & 1.55 & +1.93 & & +1.4 & +1.9 & +1.8 \\
$\mathrm{Sm}$ & 1.00 & +0.82 & & +1.2 & +0.8 & \\
$\mathrm{Gd}$ & 1.12 & +1.27 & & +1.8 & & +1.7 \\
\hline$\xi_{\text {turb }}$ & & 5.6 & & 1.8 & 2.7 & 0.5 \\
$v \sin i$ & & 20 & & 20 & 48 & 32 \\
\hline
\end{tabular}

Note: A-HD 108561, B-HD 116657, C-HD 138213.

1- Savanov (1996) using E.W. from Smith (1972), 2-Boesgaard (1987), 3-this paper.

*- The entries are upper limits.

Table 5 lists the identified lines, their $\log g f$ and approximate theoretical equivalent widths in $\mathrm{m} \AA$ computed with the resulting abundances.

\section{1. $H D 108651$}

HD 108651 (HR 4751, DM +26 2353 , Sp.Am, $V=6.65^{\mathrm{m}}$ ) is the well-known and studied Am binary. A number of authors attempted a chemical composition determination and the most elaborated results are compared with ours in Table 4. Our microturbulent velocity, $\xi_{\text {turb }}=1.8 \mathrm{~km} \mathrm{~s}^{-1}$, is remarkably lower than those given by other authors (e.g. $5.6 \mathrm{~km} \mathrm{~s}^{-1}$ by Savanov 1996, $7.0 \mathrm{~km} \mathrm{~s}^{-1}$ Smith 1971). But although derived from a single line it is in better accordance with the typical value of $2 \mathrm{~km} \mathrm{~s}^{-1}$ for B-A stars (Lemke 1989; Adelman \& Fuhr 1985). As far as $v \sin i$ is concerned, besides the value introduced in Table 2 , various values can be found in previous papers. Bernacca et al. (1971) gives $6 \mathrm{~km} \mathrm{~s}^{-1}$, while Uesugi \& Fukuda (1982) and 
Table 5. A list of the lines identified in individual spectra. Columns A, B, C give an approximate equivalent width of a line in the synthetic spectra for HD 108651, HD 116657 and HD 138213, respectively

\begin{tabular}{|l|r|r|r|r|}
\hline$\lambda$ \& Ion & $\log g f$ & $\mathrm{~A}$ & $\mathrm{~B}$ & $\mathrm{C}$ \\
\hline 6675.260 N I & -1.98 & & & 0.6 \\
6677.305 Fe II & -1.59 & 21.3 & 13.7 & 9.7 \\
6677.759 Fe I & -2.17 & 1.6 & 0.6 & \\
6677.955 Fe I & -3.63 & 1.7 & 0.6 & \\
6677.987 Fe I & -1.42 & 64.5 & 33.3 & 25.5 \\
6678.154 He I & 0.33 & & & 0.5 \\
6678.803 Co I & -2.68 & 0.8 & & \\
6678.837 Fe II & -0.45 & 1.5 & 1.2 & 1.9 \\
6678.898 Fe II & -4.88 & 1.9 & 0.9 & 1.3 \\
6679.222 Sm II & -0.83 & 1.2 & & \\
6679.352 Ca II & -0.84 & 1.6 & 1.7 & 4.7 \\
6679.566 Si II & -1.26 & & & 0.5 \\
6679.569 Fe II & -3.12 & 3.7 & 2.1 & 3.0 \\
6679.642 C I & -2.28 & 3.3 & 3.0 & 3.4 \\
6679.748 Fe II & -0.47 & 1.5 & 1.2 & 1.8 \\
6680.123 Ni I & -1.11 & 3.6 & & \\
6680.133 Ti II & -1.85 & 6.0 & 4.1 & 4.8 \\
6680.949 C I & -2.56 & 2.8 & 2.5 & 2.8 \\
6681.199 Gd II & -1.48 & 0.9 & & \\
6681.530 Sm II & -1.19 & 0.3 & 0.1 & \\
6683.161 Si I & -2.16 & 4.7 & 2.7 & 2.7 \\
6683.970 C I & -2.15 & 4.4 & 4.0 & 4.5 \\
6684.179 Fe II & -0.65 & 1.0 & 0.8 & 1.2 \\
6685.474 Fe II & -0.72 & 0.8 & 0.7 & 1.0 \\
6685.622 N I & -1.78 & 1.0 & & 1.0 \\
6685.891 C I & -3.14 & & & 0.5 \\
6687.490 Fe I & -2.32 & 3.1 & 1.2 & 0.8 \\
6687.797 Sm II & -0.97 & 0.4 & 0.1 & \\
6688.794 C I & -2.09 & 5.0 & 4.6 & 5.2 \\
6691.021 Ca I & -0.42 & 1.4 & 1.0 & 1.3 \\
6691.325 Si I & -2.97 & 0.9 & 0.5 & 0.5 \\
6692.265 Fe I & -2.95 & 0.8 & & \\
6692.447 N I & -1.28 & 3.0 & 1.2 & 3.0 \\
6693.169 Fe II & -0.73 & & 0.5 & 0.8 \\
6693.210 Fe II & -2.67 & 2.3 & 1.6 & 2.4 \\
6693.555 Sm II & -0.37 & 2.5 & & \\
6694.721 Sm II & -1.41 & 0.2 & & \\
6696.023 Al I & -1.35 & 2.3 & 3.1 & 5.2 \\
6696.044 Si I & -1.83 & 9.9 & 6.0 & 5.8 \\
6696.185 Al I & -1.58 & & 0.5 & 0.9 \\
6696.320 Fe I & -2.04 & 2.1 & 0.9 & 0.6 \\
6696.788 Al I & -1.42 & & 0.8 & 1.3 \\
6698.673 Al I & -1.65 & 1.2 & 1.6 & 2.7 \\
\hline
\end{tabular}

Kraft (1965) list $12 \mathrm{~km} \mathrm{~s}^{-1}$. Our value of $20 \mathrm{~km} \mathrm{~s}^{-1}$ is the same as that derived by Savanov (1996). In general, the abundance pattern with medium overabundances of rare earths and a low $\mathrm{Ca} / \mathrm{Fe}$ ratio (see Sect. 4.2) satisfies the pronounced Am characteristics of this star.

\section{2. $H D 116657$}

HD 116657 (HR 5055, DM +55 ${ }^{\circ} 1598$, Sp.Am, $\left.V=3.96^{\mathrm{m}}\right)$. Even if the projected rotational velocity value, $v \sin i=$ $48 \mathrm{~km} \mathrm{~s}^{-1}$, derived by us is $12 \mathrm{~km} \mathrm{~s}^{-1}$ less than the one given in Table 2, this star has one of the highest $v \sin i$ values among those so far studied for $\mathrm{Li}$ abundance. The microturbulent velocity, $\xi_{\text {turb }}=2.7 \mathrm{~km} \mathrm{~s}^{-1}$, is not unusual for these stars. The abundance pattern, as well as the $\mathrm{Ca} / \mathrm{Fe}$ ratio, aligns the star with typical Am stars.
Table 5. continued

\begin{tabular}{|l|r|r|r|r|}
\hline$\lambda$ \& Ion & $\log g f$ & $\mathrm{~A}$ & $\mathrm{~B}$ & $\mathrm{C}$ \\
\hline 6699.142 Fe I & -2.10 & 2.6 & 1.1 & 0.7 \\
6699.164 Fe II & -4.04 & & & 0.6 \\
6700.890 Ni I & -2.32 & 1.0 & & \\
6702.862 N I & -1.81 & 0.9 & & 0.9 \\
6703.567 Fe I & -3.16 & 3.5 & 1.1 & 0.9 \\
6704.147 GdII & -1.83 & 0.3 & & 0.1 \\
6704.481 Fe I & -2.66 & 1.3 & 0.5 & \\
6704.524 Ce II & -0.51 & & 1.2 & 0.9 \\
6704.839 N I & -1.35 & 2.6 & 1.0 & 2.6 \\
6705.101 Fe I & -1.10 & 16.4 & 7.3 & 5.0 \\
6705.131 Fe I & -2.37 & 0.8 & & \\
6706.051 Ce II & -1.25 & & 0.5 & \\
6706.107 N I & -1.80 & 0.9 & & 0.9 \\
6706.880 Fe II & -4.10 & & & 0.5 \\
6706.980 Si I & -2.48 & 2.9 & 1.7 & 1.7 \\
6707.473 SmII & -1.48 & 0.3 & 0.1 & \\
6707.761 Li I & -0.01 & 6.0 & 1.7 & 2.6 \\
6707.912 Li I & -0.31 & 3.1 & 0.9 & 1.3 \\
6708.759 N I & -1.79 & 1.0 & & 1.0 \\
6708.885 Fe II & -0.52 & 1.4 & 1.1 & 1.6 \\
6711.323 C I & -2.47 & 3.4 & 3.0 & 3.4 \\
6711.575 Ni I & -3.81 & 1.0 & & \\
6712.438 Fe I & -2.16 & 1.3 & 0.5 & \\
6712.676 Fe I & -4.62 & 0.2 & 0.1 & 0.0 \\
6713.046 Fe I & -1.61 & 6.8 & 2.9 & 2.0 \\
6713.195 Fe I & -2.56 & 1.8 & 0.7 & 0.5 \\
6713.586 C I & -2.17 & 6.6 & 5.9 & 6.6 \\
6713.771 Fe I & -1.60 & 5.9 & 2.5 & 1.7 \\
6715.383 Fe I & -1.64 & 7.0 & 2.9 & 2.0 \\
6716.237 Fe I & -1.92 & 4.0 & 1.6 & 1.1 \\
6716.973 Si II & -0.10 & & & 0.7 \\
6717.298 Fe I & -1.96 & 1.8 & 0.7 & 0.5 \\
6717.524 Fe I & -2.45 & 1.2 & 0.5 & \\
6717.681 Ca I & -0.61 & 16.1 & 10.4 & 12.7 \\
6717.794 Ti II & -1.80 & 6.4 & 4.4 & 5.2 \\
6717.964 Fe II & -0.81 & & & 0.7 \\
6718.130 Gd II & -1.00 & 0.7 & & 0.3 \\
6718.883 Fe II & -0.73 & & & 0.7 \\
6719.609 Si I & -2.50 & 2.0 & 1.1 & 1.1 \\
6719.639 Fe II & -0.52 & 1.3 & 1.0 & 1.6 \\
6720.280 Ce II & -1.35 & & 0.5 & \\
6720.908 Si I & -2.41 & 3.9 & 2.2 & 2.2 \\
6721.848 Si I & -1.49 & 26.9 & 16.1 & 15.3 \\
6722.077 Fe II & -0.57 & 1.0 & 0.8 & 1.2 \\
6722.610 N I & -0.98 & 5.8 & 2.4 & 5.9 \\
6722.759 Co I & -0.81 & 1.3 & & \\
6723.220 Co I & -0.99 & 0.8 & & \\
\hline
\end{tabular}

\section{3. $H D 138213$}

HD $138213\left(\right.$ HR 5752, DM +47 2227 , Sp.Am, $\left.V=5.96^{\mathrm{m}}\right)$. The value of the projected rotational velocity, $v \sin i=$ $32 \mathrm{kms}^{-1}$, estimated by us is close to the one given in Table 2. The microturbulence derived, following the procedure described, corresponds to $\xi_{\text {turb }}=0.5 \mathrm{~km} \mathrm{~s}^{-1}$. Considering the lower surface gravity, $\log g=3.6$, this is rather a low value. The Am anomalies of this star are not as pronounced as in the two other stars and the $\mathrm{Ca} / \mathrm{Fe}$ ratio is larger. If its true rotational velocity was smaller than that of HD 116657, which is more peculiar, this could be an interesting paradox. The mild anomalies could be a result of its lower gravity, $\log g=3.6$ as evolved stars might loose their Ca deficiency (Berthet 1992) and/or rotationally induced mixing might be more effective in such stars (Michaud 1982). 


\section{Discussion}

\subsection{Abundance pattern of program stars}

Below we discuss the abundance pattern of the stars studied (consult Table 4 for a summary).

Lithium - as derived from two lines at $\lambda 6707.761$ and $\lambda 6707.912$ the abundance is normal, compared with normal A-type stars, in HD 108651 and HD 138213 and slightly underabundant in HD 116657 (see Fig.1). While in the spectrum of HD 108651 the lithium lines dominate the absorption feature observed, in the two hotter stars Li lines weaken and unidentified opacity occurs on the long-wave side of this feature at about $\lambda 6708.6 \pm 0.3$. This opacity could not be explained by means of adjusting the $\log g f$ value of Fe II $6708.885 \AA$ as increasing its $\log g f$ would have lead to unacceptable strengthening of the line in the spectrum of the cooler star. Recently, Hack et al. (1997) also pointed to a similar lack of opacity in the atomic data at $\lambda 6708.9$ in $\beta \mathrm{CrB}$. Thus, the Li abundance estimated for the two hotter stars is rather an upper limit.

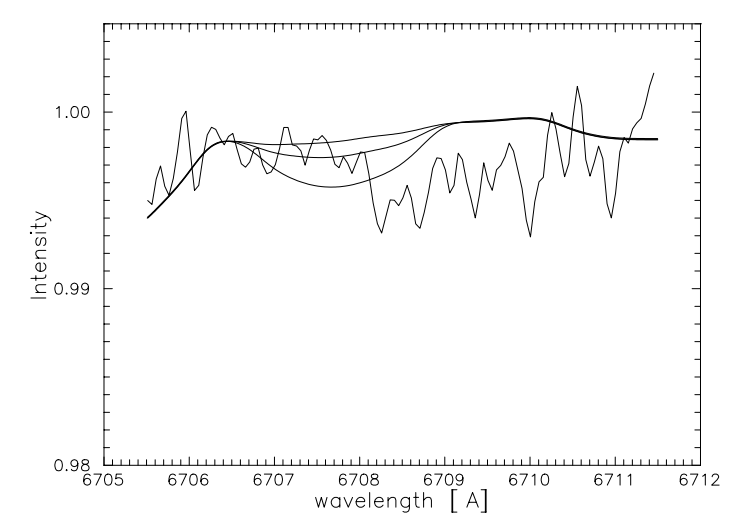

Fig. 1. A close look at the Li 6708 region in HD 116657. Synthetic spectrum computations were made with the following abundances $\log N(\mathrm{Li}) / H+12=2.50,2.80,3.10$ (from top to bottom)

Carbon - the abundance of carbon can be derived from two lines: C I 6683.970 which includes a slight blend of FeII, and C $\mathrm{C}_{\mathrm{I}} 6688.794$ which stands alone. Three other $\mathrm{C}_{\mathrm{I}}$ lines are only complements of blends. One line, $6711.323 \AA$, though not-negligible in the computed spectra, is not present in those observed. A mild deficit of carbon in all three stars favors Am characteristics.

Nitrogen - seven lines could be identified in blends, but only one of them, $6722.610 \AA$, makes a significant contribution to the wing of Si I 6721.848 . This enabled us to estimate nitrogen abundance. While normal in HD 116657, nitrogen was slightly overabundant in the two other stars.

Aluminium - the lines of $\mathrm{Al}$ I significantly contribute to the absorption at $6696 \AA$ and $6699 \AA$. Aluminium is moderately and slightly underabundant in HD 108651 and HD 116657 respectively, and is normal in HD 138213.
Silicon - Si I 6721.848 causes a substantial absorption at the feature observed, which enables us to derive its abundance with accuracy. The other lines, 6683.161, $6696.044,6706.980,6719.609$ and $6720.908 \AA$, occurring alone or in well-defined wings, confirm the derived value. Silicon is slightly to moderately overabundant in all three stars.

Calcium-Ca I 6717.681 dominates one of the strongest features in the observed spectra. Ca II 6679.352 complements the blend in the long-wave wing of the strongest Fe I line. Due to an unidentified opacity source within Ca I 6718 the abundance given in Table 4 is rather an upper limit as shown in Sect. 4.2. Nevertheless, the abundances derived confirm at least a slight calcium deficit in HD 108651 and HD 116657, while in HD 138213 the abundance is normal at most. The calcium deficit underlines the Am characteristics of those two stars.

Titanium - is normal as estimated from Ti II 6680.133 which contributes significantly to the blend on the wing of the strongest Fe I line.

Iron - the procedure for deriving iron abundance was described in the previous section. Its abundance correlates with the other approximately $35 \mathrm{Fe}$ I and Fe II lines. Iron might be slightly overabundant in HD 108651, while it is normal in the two other stars.

Nickel - only in HD 108651 are two lines identified with Ni I 6680.123 and Ni I 6700.890. The former Ni line contributes to Ti II 6680.133 and suggests a moderate overabundance. There is a minute depression in the noise at this place in Ti I line. The third Ni I line at $6711.575 \AA$ in the observed spectrum is unsignificant. Thus, the value estimated represents rather an upper limit of the abundance.

Cerium, Samarium, Gadolinium - three lines of cerium, 6 of samarium and 3 of gadolinium are suitable minor complements of blends with other lines. The values derived are rather upper limits of the abundances.

\subsection{Ca/Fe ratio and lithium in binary systems with an Am component}

The following analysis is based on data from Boesgaard (1987), Burkhart \& Coupry $(1989,1991,1997)$ and this paper as far as the $\mathrm{Ca} / \mathrm{Fe}$ (the ratio of measured equivalent widths of $\mathrm{Ca}$ I 6718 and Fe I 6678 blends, i.e. comprising also e.g. Fe II 6677 line) and $\mathrm{Li}$ is concerned, and from Batten et al. (1989) as to the orbital elements. Consequently, one should be aware of the disadvantages of such data compilations from various sources, e.g. the sample is not homogeneous.

Note that the only observed Ca I 6718 line blends with a strong Ti II 6718 line, as recently mentioned by Burkhart \& Coupry (1989). Unfortunately, there remains a lack of opacity in the atomic data so that one cannot derive reliable Ca abundance from this line. This follows from Fig. 2, where we plot $\mathrm{Ca} / \mathrm{Fe}$ against $T_{\text {eff }}$. The dashed line is the 
simulated synthetic $\mathrm{Ca} / \mathrm{Fe}$ ratio calculated for solar abundances with $\log g=4.0$. One can see that it is apparently less than the one observed for normal stars, pointing to the mentioned missing opacity. Nevertheless, one can see that the normal and Am stars are well separated in such a plot and that the $\mathrm{Ca} / \mathrm{Fe}$ ratio in normal stars is not very sensitive to their $T_{\text {eff }}$ and is mainly within $0.8-1.0$. Such scatter is well in accordance with the expected uncertainty in the equivalent width measurement. The latter is usually $\lesssim 10 \%$ so that the observed relative error in the $\mathrm{Ca} / \mathrm{Fe}$ ratio is $\lesssim 20 \%$. We can see that three out of the four long period Am binaries clearly occupy the bottom of Fig. 2 (see also Table 6). This is a strong indication of their pronounced Am anomalies.

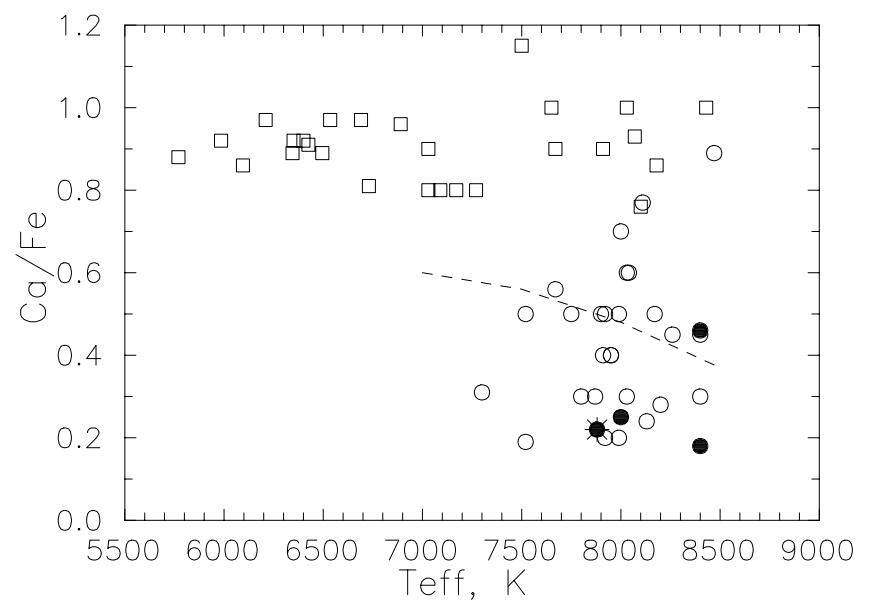

Fig. 2. Ca/Fe ratio versus $T_{\text {eff }}$. Data are from Boesgaard (1987), Burkhart \& Coupry $(1989,1991,1997)$ and this paper. Squares stand for normal stars; open circles for Am stars; full circles for our long period Am binaries and 16 Ori (which is moreover distinguished also by an asterix); dashed line is calculated $\mathrm{Ca} / \mathrm{Fe}$ behaviour for solar abundances

Table 6. Measured equivalent width of Ca I 6718 and Fe I 6678 in $\mathrm{m} \AA$

\begin{tabular}{|l|l|l|l|}
\hline & HD 108561 & HD 116657 & HD 138213 \\
\hline Ca I & 23.7 & 13.0 & 25.6 \\
Fe I & 95 & $73^{*}$ & 56 \\
$\mathrm{Ca} / \mathrm{Fe}$ & 0.25 & 0.18 & 0.46 \\
\hline
\end{tabular}

Note: * - including the Tirl 6680 blend in the red wing of FeI 6678.

We proceed to explore any possible dependence of $\mathrm{Ca} / \mathrm{Fe}$ on orbital parameters. There might be a decrease of $\mathrm{Ca} / \mathrm{Fe}$ with the orbital period, but it is not convincing (Fig. 3a). Apparently, HD 138213 does not fit into such a pattern having $\mathrm{Ca} / \mathrm{Fe}=0.46$. However, notice that it is a special case of long period binaries (with $P_{\text {orb }}<200^{\mathrm{d}}$ ) having a circular orbit. Such stars may not obey the favoured behaviour of increasing metallicity with $P_{\text {orb }}$ (Budaj 1997). Nevertheless, looking at Fig. 3b, we see a remarkable decline of $\mathrm{Ca} / \mathrm{Fe}$ with eccentricity. To speak in more objective terms, we have calculated Pearson's linear $\left(r_{\mathrm{l}}\right)$ as well as Spearman's rank order $\left(r_{\mathrm{s}}\right)$ correlation coefficients together with their two-sided significances or p-values $\left(p_{\mathrm{l}}, p_{\mathrm{s}}\right.$, according to Press et al. 1986). The latter simply represent the probability of the appearance of a better correlation coefficient than that found here under the assumption that the quantities do not correlate at all. Generally, a $p$-value less than about 0.05 is accepted as strong support for the presence of a correlation. We see in Table 7 that there is sufficient evidence that $\mathrm{Ca} / \mathrm{Fe}$ decreases with eccentricity.

Table 7. Correlation coefficients for the relation of $\mathrm{Ca} / \mathrm{Fe}$ and $\mathrm{Li}$ with $\log P_{\text {orb }}$ and eccentricity together with their corresponding significances

\begin{tabular}{|l|l|l|l|l|}
\hline \multicolumn{3}{|c|}{$\mathrm{Ca} / \mathrm{Fe} \times$} & \multicolumn{2}{c|}{$\mathrm{Li} \times$} \\
& $\times e$ & $\times \log P_{\text {orb }}$ & $\times e$ & $\times \log P_{\text {orb }}$ \\
\hline$r_{1}$ & -0.51 & -0.39 & -0.64 & -0.40 \\
$p_{1}$ & 0.036 & 0.12 & 0.019 & 0.18 \\
$r_{\mathrm{s}}$ & -0.63 & -0.57 & -0.95 & -0.46 \\
$p_{\mathrm{s}}$ & 0.007 & 0.16 & $710^{-7}$ & 0.12 \\
\hline
\end{tabular}

Similar analysis was also performed in the case of $\mathrm{Li}$ (see Figs. 3c and 3d). Typical errors of most Li abundances are mainly due to the uncertainties in effective temperatures and amount to about 0.2 dex (Burkhart \& Coupry 1989). We also observe here a tendency of $\mathrm{Li}$ abundance to decrease with orbital period, but it is again not very convincing. However, there is sufficient evidence that $\mathrm{Li}$ declines with eccentricity (check Table 7 for corresponding significances). This strongly supports our above findings concerning the $\mathrm{Ca} / \mathrm{Fe}$ behaviour because the anomalies in both quantities behave in the same manner, namely, they increase with rising eccentricity ${ }^{2}$ and exhibit a less pronounced but similar tendency with orbital period. Unfortunately, it is hard to distinguish from such sparse data whether the latter behaviour is not affected by the former, or the opposite, because in general there is a predominance of eccentric orbits at large periods. Nevertheless, this cannot be a mere coincidence. Rather, it is exactly the opposite of what has recently been found in the peculiarity of Ap binaries by Budaj et al. (1996) (see also Gerbaldi et al. 1985; Budaj 1995), namely, that Ap peculiarity diminishes at larger eccentricities. This means

$\overline{2}$ To state it more exactly pronounced anomalies seem more
frequent than mild ones at larger eccentricities while at low ec-
centricities both mild and pronounced anomalies are common. 

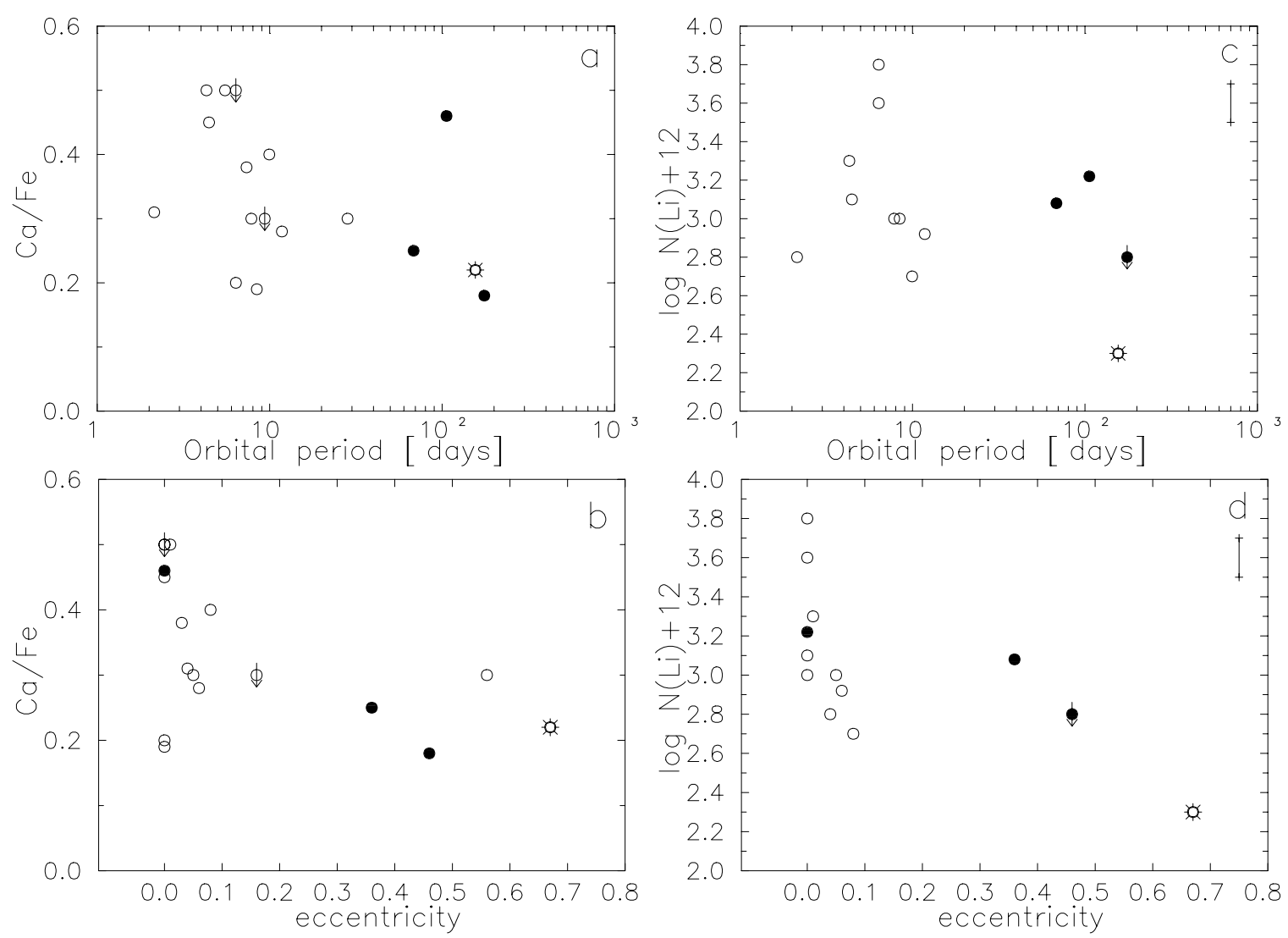

Fig. 3. Ca/Fe ratio and $\mathrm{Li}$ versus eccentricity and $P_{\text {orb }}$. Open circles stand for Am binaries from Boesgaard (1987) and Burkhart \& Coupry $(1989,1991,1997)$ (16 Ori is moreover distinguished by a special symbol); full circles are used for our long period Am binaries; arrows denote upper limits. Typical error in $\mathrm{Ca} / \mathrm{Fe}$ is about $20 \%$ and in Li abundance about 0.2 dex (displayed in the top right-hand corner of $3 \mathrm{c}, \mathrm{d}$ )

that apart from (1) orbital periods and (2) frequency of occurrence among SB2's (Abt \& Snowden 1973; Abt \& Levy 1985) these two basic subgroups of CP stars are furthermore distinguished by their (3) eccentricities. This supports the idea that there is a basic set of parameters connected with binarity which determines CP characteristics. Such eccentricity effects could be accounted for by a different degree of pseudo-synchronization, which is apparently much higher for eccentric than for circular orbits at comparable orbital periods. Following Budaj (1997) and Budaj et al. (1996) this could not only lower the efficiency of tidal mixing in corresponding Am binaries ${ }^{3}$, but have a much more serious impact on stellar magnetism, for example... Thus, based on the observed decline of $\mathrm{Li}$ with eccentricity (or its marginal decline with $P_{\text {orb }}$ ), a potential Li deficit in HD 116657, and the above-mentioned relationship between binarity and CP phenomena we propose that the 16 Ori phenomenon might be due to the suppressed tidal mixing. The latter, being more intensive in low eccentric or short period orbits, suppresses diffusion

\footnotetext{
$3 \quad$ Also because eccentric orbits generally exhibit a larger $P_{\text {orb }}$ and tidal effects weaken with increasing separation between the components.
}

and disperses a potential $\mathrm{Li}$ cloud as well as any other abundance anomalies (including $\mathrm{Ca} / \mathrm{Fe}$ ) below the superficial convection zone. This is not the case with 16 Ori or HD 116657, which, having the longest $P_{\text {orb }}$ (within the range $P_{\text {orb }}<200^{\mathrm{d}}$ ), and large eccentricities, could build relatively pronounced abundance anomalies in their stable atmospheres.

\section{Conclusion}

The $\mathrm{Ca} / \mathrm{Fe}$ ratio, a suitable peculiarity indicator, was found to be quite low for three out of four Am binaries within $50^{\mathrm{d}}<\log P_{\text {orb }}<200^{\mathrm{d}}$. We revealed sufficient evidence that this ratio declines (i.e. the Am anomaly increases) with increasing eccentricity. Such behaviour of the Am peculiarity acquires a firmer footing when complemented by the behaviour of lithium abundances, which also exhibit an analogous increase in their anomalies with eccentricity. We found HD 116657 to be another candidate as a $\mathrm{Li}$ deficient star among long period Am binaries, in addition to 16 Ori. To summarize, the main results met our expectation that long-period Am binaries $\left(50^{\mathrm{d}}<\log P_{\text {orb }}<200^{\mathrm{d}}\right)$ exhibit relatively large 


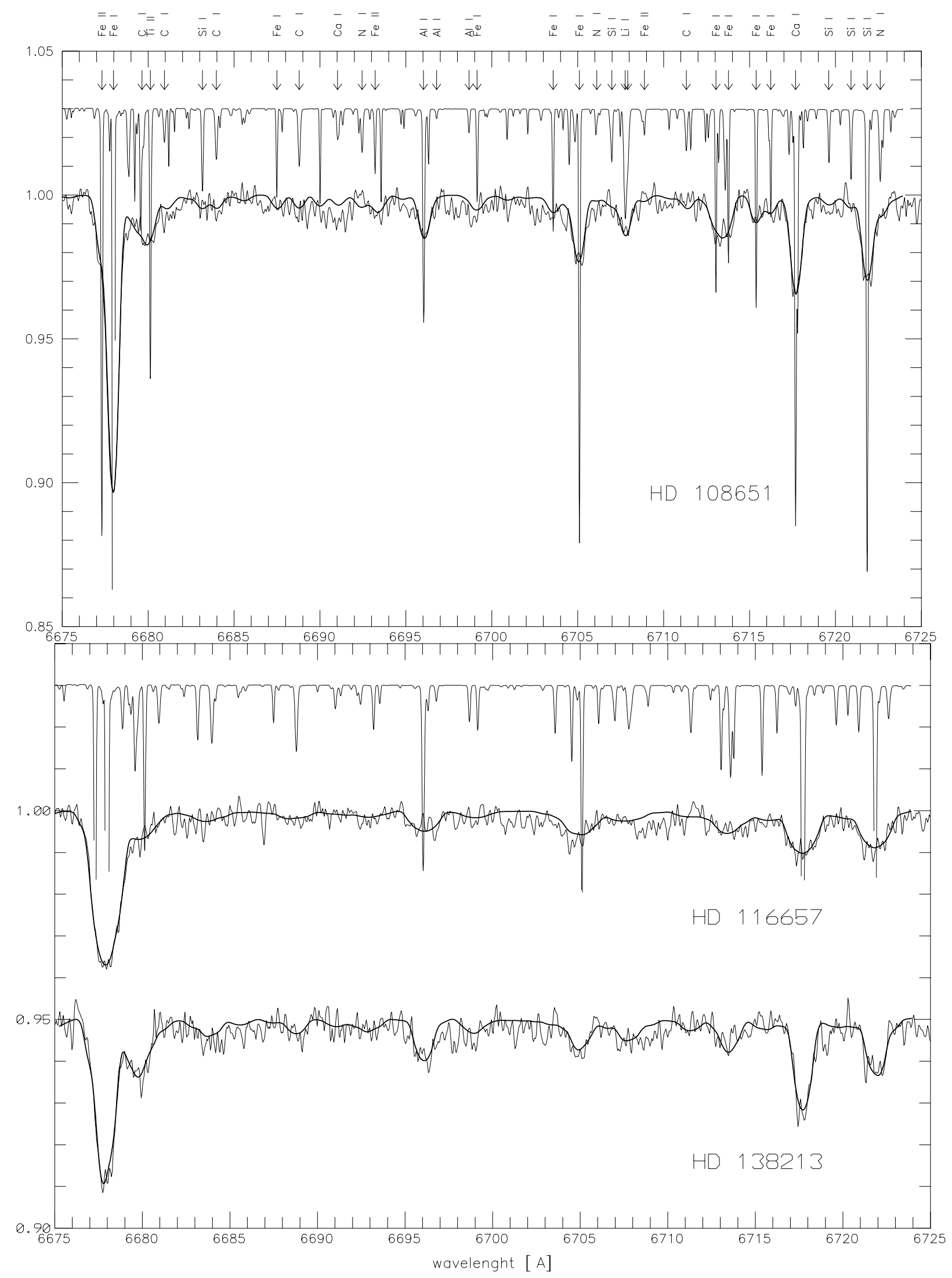

Fig. 4. Spectrum synthesis of the region $6675-6725 \AA \AA$ of the program stars. Model atmosphere parameters are from Table 3. The derived abundances are listed in Table 4. The synthetic "non-rotated" spectra are for HD 108651 and HD 116657 and they are cut below 0.85 and 0.98 , respectively 
abundance anomalies when compared with other Am stars. However, at present, it is beyond our spectroscopic and statistical data to determine whether a possible marginal $P_{\text {orb }}$ dependence could be real, or whether it is due to the more pronounced eccentricity relationship because of the general predominance of eccentric orbits at larger periods. Nevertheless, this emphasizes that tidal effects are of crucial importance and no account on Am binaries is complete in which their role is reduced to being simply a tool to slow down the rotation below some cutoff velocity. An $\mathrm{S} / \mathrm{N}$ more than $\approx 1000$ in the lithium region, a lager sample of stars treated by the same method and reliable $\mathrm{Ca}(\mathrm{C}, \mathrm{Mg}, \mathrm{Sc})$ abundance data are required to pursue the problem further.

Acknowledgements. This study made use of the Vienna Atomic Line Database (VALD) services. The authors acknowledge the support of VEGA grants Nos. 1002 and 4175. I.I. and I.B. acknowledge the support of the Ministry of Science and Education grant F-603 and CEEPUS project BG-2. We also wish to thank G.A. Wade and P. Hunt for proofreading the paper, R. Komžík for his care of the computer facilities at our institute, as well as to the referee Dr. C. Van't Veer for extremely helpful and constructive comments.

\section{References}

Abt H.A., 1961, ApJS 6, 37

Abt H.A., 1965, ApJS 11, 429

Abt H.A., Bidelman W.P., 1969, ApJ 158, 1091

Abt H.A., Levy S.G., 1985, ApJS 59, 229

Abt H.A., Snowden M.S., 1973, ApJS 25, 137

Adelman S.J., Fuhr J.R., 1985, A\&A 152, 434

Batten A.H., Fletcher J.M., MacCarthy D.G., 1989, Eight Catalogue of Orbital Elements of Spectroscopic Binary Systems, Publ. Dominion Astrophys. Obs. Vol. 17

Bernacca P.L., Perinotto M., 1971, Contr. Oss. Astrofis. Univ. Padova 249

Berthet S., 1992, A\&A 253, 451

Boesgaard A.N., 1987, ApJ 321, 967

Budaj J., 1994, poster at JD12, 22nd General Assembly, The Hague

Budaj J., 1995, in: Stellar Surface Structure - Poster Proceedings from IAU Symp. 176, Strassmeier K.G. (ed.), p. 147

Budaj J., 1996, A\&A 313, 523

Budaj J., 1997, A\&A (accepted)

Budaj J., Elkin V.G., Romanyuk I.I., Wade G.A., Žižňovský J., Zverko J., 1997, in Stellar Magnetic Fields, Glagolevskij Yu.V. and Romanyuk I.I. (eds.), Moscow (in press), p. 176 Burkhart C., Coupry M.F., 1989, A\&A 220, 197

Burkhart C., Coupry M.F., 1991, A\&A 249, 205

Burkhart C., Coupry M.F., 1997, A\&A 318, 870

Burkhart C., Coupry M.F., Lunel M., van’t Veer C., 1987,
A\&A 172,257

Castelli F., Kurucz R.L., 1994, poster at JD12, 22nd General Assembly, The Hague

Conti P.S., Barker P.K., 1973, ApJ 186, 185

Gerbaldi M., Floquet M., Hauck B., 1985, A\&A 146, 341

Gerbaldi M., Faraggiana R., Castelli F., 1995, A\&AS 111, 1

Grevesse N., Anders E., 1991, in: Solar Interior and Atmosphere, Cox A.N., Livingston W.C., Matthews M.S. (eds.). Univ. of Arizona press, Tuscon

Gutmann F., 1965, P. Dom. Ap. O. 12, 361

Hack M., Polosukhina N.S., Malanushenko V.P., Castelli F., 1997, A\&A 319, 637

Hauck B., Künzli M., 1996, Baltic Astron. 5, 303

Hill G., Adelman S.J., 1986, in Upper Main Sequence Stars with Anomalous Abundances, Cowley C.R., Dworetsky M.M., Megessier C. (eds.), p. 209

Hubený I., 1987, Scient. Tech. Rep. Astron. Inst. Czechoslov. Acad. Sci. 40

Iliev I.Kh., Budaj J., 1996, in Proceedings of MASS workshop, Vienna, ASP Conf. Proc. 108 (in press)

Kobi D., North P., 1990, A\&AS 85, 999

Kraft R.P., 1965, ApJ 142, 681

Kurucz R.L., 1992, Rev. Mexicana Astron. Af. 23, 45

Kurucz R.L., Furenlid I., Brault J., Testerman L., 1984, Solar Flux Atlas from 296 to $1300 \mathrm{~nm}$, Harvard University

Lemke M., 1989, A\&A 225, 125

Lucy L.B., Sweeney M.A., 1971, AJ 76, 544

Michaud G., 1982, ApJ 258, 349

Moon T.T., Dworetsky M.M., 1985, MNRAS 217, 305

Napiwotzky R., Schönberner D., Wenske V., 1993, A\&A 268, 653

Piskunov N.E., 1992, in Stellar Magnetism, Glagolevskij Yu.V. and Romanyuk I.I. (eds.). St.-Petersburg, Nauka, p. 92

Piskunov N.E., Kupka F., Ryabchikova T.A., Weiss W.W., Jeffery C.S., 1995, A\&AS 112, 525

Press W.H., Flannery B.P., Teukolsky S.A., Vetterling W.T., 1986, Numerical Recipes. Cambridge Univ. Press, Cambridge

Renson P., Kobi D., North P., 1991, A\&AS 89, 61

Renson P., 1991, Catalogue Général des Etoiles Ap et Am, Astr. Inst.-Univ. de Liège

Richer J., Michaud G., 1993, ApJ 416, 312

Rufener F., 1980, Third Catalogue of Stars Measured in the Geneva Obs. Phot. System, Obs. de Genève

Savanov I.S., 1996, AZh 73, 221

Smalley B., Dworetsky M.M., 1995, A\&A 293, 446

Smirnov O.M., Piskunov N.E., Afanasyev V.P., Morozov A.I., 1992, in Astronomical Data Analysis Software and Systems, I, Worrall D.M., Biemsdorfer C. and Barnes J. (eds.), ASP Conf. Ser. 26. San Francisco, ASP, p. 344

Smith M.A., 1971, A\&A 11, 325

Smith M.A., 1972, A\&AS 5, 81

Tassoul J.-L., Tassoul M., 1992, ApJ 395, 259

Uesugi A., Fukuda I., 1982, Revised Catalogue of Stellar Rotational Velocities, Depart. of Astron. Kyoto University 